\title{
A Geographical Factor of Interest Recommended Strategies in Location Based Social Networks
}

\author{
Bulusu Rama $^{*}$, K. Sai Prasad ${ }^{2}$, Ayesha Sultana ${ }^{3}$, K. Shekar ${ }^{4}$ \\ ${ }^{I}$ Department of Computer Science and Engineering, MLR Institute of Technology, Dundigal, Hyderabad, India. \\ ${ }^{2}$ Department of Computer Science and Engineering, MLR Institute of Technology, Dundigal, Hyderabad, India. \\ ${ }^{3}$ Department of Computer Science and Engineering, MLR Institute of Technology, Dundigal, Hyderabad, India. \\ ${ }^{4}$ Department of Computer Science and Engineering, MLR Institute of Technology, Dundigal, Hyderabad, India. \\ *Corresponding author E-mail:bulusurama1967@gmail.com
}

\begin{abstract}
The fast development of area based administrations (LBSNs) has extensively advanced individuals' city lives and pulled in a huge number of recent years. Area based informal organizations (LBSNs) allow clients to registration at a real region and offer step by step rules on purposes of-intrigue (POIs) with their pals each time and anyplace. Such check-in behavior can make daily real-life experiences spread rapidly via the Internet. Moreover, such check-in records in LBSNs can be totally exploited to understand the basic legal guidelines of humans' every day motion and mobility. This paper centers on evaluating the scientific classification of client displaying for POI proposals through the information investigation of LBSNs. First, we quickly introduce the shape and records traits of LBSNs, then we current a formalization of user modeling for POI suggestions in LBSNs. Contingent upon which sort of LBSNs records used to be completely used in buyer displaying forms for POI proposals, we separate client demonstrating calculations into four classifications: pure check-in data-based consumer modeling, geographical information-based consumer modeling, spatial-temporal information-based consumer modeling, and geo-social information-based consumer modeling. At finally, condensing the current works, we bring up the future difficulties and new guidelines in five possible aspects
\end{abstract}

Keywords: Point of interest, social networks, location-based services.

\section{Introduction}

The superior facts technologies that have resulted from the speedy increase of location-based services (LBSs) have notably enriched people's city lives. Area based interpersonal organizations (LBSNs) allow clients to landing and offer their areas, tips, and encounters in regards to purposes of-intrigue (POIs) with their amigos whenever and anyplace. Such registration lead can make genuine every day encounters spread rapidly finished the Internet. In addition, such registration information of LBSNs can be completely misused to perceive the essential laws of human day by day development and versatility [1], which can be used to proposal structures and area based administrations. Therefore, area based online networking certainties administrations are drawing in noteworthy consideration from one of kind trade areas e.g. purchaser profiling, proposal frameworks, city crisis competition assertion, urban arranging and promoting decisions. User produced spatial-fleeting records can be amassed from LBSNs and can be generally utilized for discernment and demonstrating human mobility as indicated by the accompanying 4 viewpoints:

\subsection{Geographical Feature}

The spatial factors regarding social movement as like hidden between lots of on thousands of arrival knowledge has been exploited in accordance with acknowledge ethnic quality. as an example, social beings have a bent in keeping with move when close places and each consequently repeatedly in accordance with remote locations the previous is short-ranged expertise or isn't any longer suffering from means that of the utilization of social community ties, topical each spatially or temporally; the trendy is longdistance cowl and a lot of influenced by communal neighborhood ties.

\subsection{Temporal Features}

The routines and habits of our everyday lives, there are unique possibilities for distinct areas at particular hour of the day and particular week of the days the check in data of LBSN likewise reveals these results. On the finishes of the week, most registration practices happen toward beginning of the day or evening, and the zones are closed to specific POIs (e.g. a market, museum, and restaurant).

\subsection{Social Features}

First, many lookup show that humans tend to go to shut areas extra often than faraway places, however they have a tendency to go to far-off areas shut to their friends' residences or these that are checked-in through their friends. These observations have been greatly used for vicinity suggestions in LBSNs. Second, the spatial-worldly capacity dreamy from registration records has been abused to deduce social ties and buddy suggestions. 


\subsection{Integrated Feature}

As one sort of worldwide open information source about character movement related decisions, the registration data in LBSNs gives another approach to detect individuals' spatial and fleeting inclinations and gather their social ties.

\section{Characteristics of Lbsns}

\subsection{Structure of Lbsns}

LBSNs are primarily based totally on frequent on-line social networks and provide location-based services that enable customers to check-in at bodily areas and share location-related statistics with their friends. Meanwhile, LBSNs supply a new point of view for crossing over any barrier between the genuine and computerized universes that allow clients' genuine topographical activities to be scattered on the Internet.
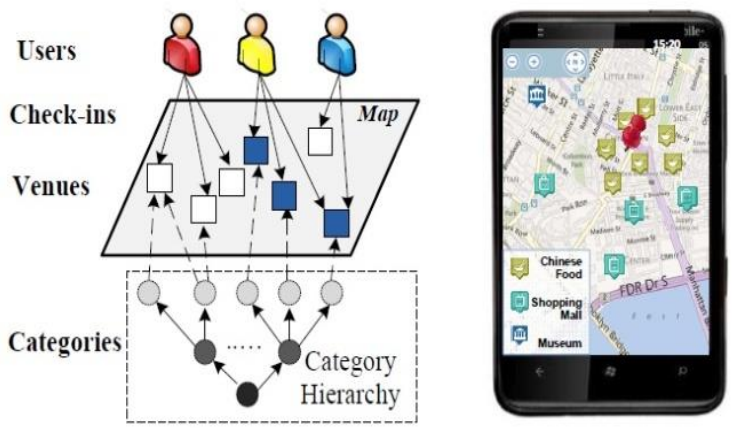

Fig. 1: [1-1.2] Location-based and preference-aware recommendations using sparse check-in data

Customarily, area systems, client area systems, and area media systems are the key research content and most clients displaying for POI pointers in LBSNs are composed from the factor of records mining and looking at these three systems.

\subsection{The Archives Characteristics of Lbsns}

There are three sorts of data in LBSNs:

1. User check-ins: the information files customer's checkins at tremendous geographical locations at one of a variety times.

2. Users social relationships: the statistics files users' social relationships.

3. Social activities: the statistics files the social things to do the vicinity clients take section at exceptional geographical locations and at one of kind times, or shared social media information.

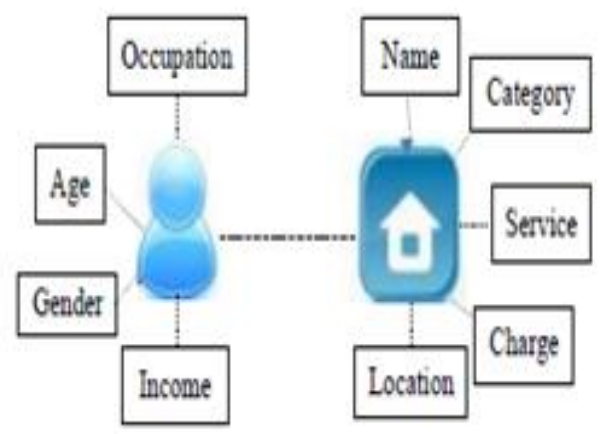

Fig. 2: The structure of user-POI data

\section{Geographical Information-Based User Modeling}

Like common recommender systems, the above-mentioned methods regularly deal with POIs as items; however do no longer consider geographical influence, which is a unique attribute that distinguishes POIs from gadgets in regular recommender systems. Therefore, leveraging the geographical information of users' check-ins (as shown in Fig.3) can seize the spatial distribution of humans' daily motion and enhance the performance of POI recommendation systems in LBSN.

\subsection{Bayesian Model-Based Consumer Modeling}

Similar to Fig. 3, much research [19-22] have shown that the spatial clustering phenomenon of users' check-ins in LBSNs, which results from users' tendency to go to nearby places as an alternative than distant ones in their everyday lives. It is intuitive that the Bayesian mannequin [20] and probabilistic approach can be employed to mannequin the geographical affect of person check-ins in LBSNs. For example, to mannequin the geographical influence of users' check-in behaviors, [20] utilized the electricity regulation distribution to mannequin the geographical influence among POIs and proposed a collaborative POI suggestion algorithm that was based on a naïve Bayesian one. [22] Proposed a probabilistic method to model customized geographical influence on consumer check-in behavior and predict the likelihood of a consumer travelling a new location. To mannequin the numbers of centers that are checked-in with the aid of distinct users' LBSNs [18] computed the probability of a consumer checking in to a area through a multi-center Gaussian model and proposed a POI recommendation framework with a mixture of consumer preference, geographical influence, and personalized ranking. [19] Proposed an out-of-town place advice algorithm in consideration of the spatial have an effect on between POIs to measure a region's attractiveness. By taking the spatial have an effect on of users' check-ins into account, it should slim the looking out house to beautify the performance of suggestion systems. The disadvantage of these processes is that they ought to no longer deal with person cold-start problem.

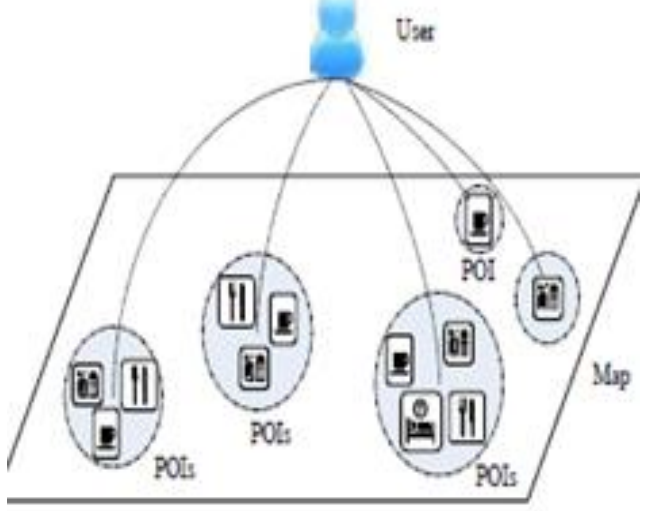

Fig. 3: Spatial cluster of user check-ins

\subsection{Latent Issue Model}

Alongside the improvement of the matrix factorization approach in the recommendation system, any other intuitive approach of modeling customers based on geographical statistics in LBSNs is the latent aspect model. The most important undertaking is how to mix the geographical impact two of user two behaviors matrix factorization. In general, the inherent spatial characteristic (e.g. neighbor) of POIs and the spatial clustering two phenomenons (e.g. two all customers who go to POIs have a tendency to cluster together and several geographical areas are robotically formed) are the core geographical influences that are regarded in the latent element model, and are factorization. In general, the inherent spa- 
tial feature (e.g. neighbor) of POIs and the spatial cluster two phenomenon's (e.g. All users two who visit POIs have a tendency to cluster together and a number of generally treated as extra latent elements in matrix factorization. The two present day strategies to consumer modeling can be divided into two groups.

\subsubsection{Geographical Neighbor}

The observations that humans tend to visit nearby POIs and their geographical neighbors in LBSNs have been correctly used in POI recommendations. The envisioned ranking and objective characteristic were as follows.

$$
\begin{gathered}
\hat{r}_{u i}=\mu+b_{u}+b_{i}+z p_{u}^{r}\left(1 /|R i| \sum_{w \in R_{i}} q_{w}+\alpha_{1} /\left|N_{i}\right| \sum_{n \in N_{i}} V_{n}\right. \\
\left.+\alpha_{2} /\left|C_{i}\right| \sum_{c \in C_{i}} d_{C}\right)
\end{gathered}
$$$$
\min _{p *, q *, b *, v *, d *, \beta *,(U, i) \in K} \sum\left(r_{u i-} \hat{r}_{u i)}+\right.
$$$$
\left.\lambda_{1}\left(\left\|P_{u}\right\|^{2}\right)+\sum_{w \in R_{i}}\left\|q_{w}\right\|^{2}\right)+\lambda_{2}\left(b_{u}^{2}+b_{i}^{2}+\beta_{i}^{2}+\right.
$$$$
\left.\beta_{u}^{2}\right)+\lambda_{3}\left(\sum_{n \in N_{i}}\left\|V_{n}\right\|^{2}+\sum_{c \in C_{i}}\left\|d_{c}\right\|^{2}\right)
$$

Where $\mu$ is the common ranking of all recognized ratings, $b_{u} \mathrm{u} b$ and $b_{i}$ signify the person bias and item bias, $p_{u}$ represents the latent elements of user $\mathrm{u}, p_{i}$ represents the latent factors of object $\mathrm{i}$ for its intrinsic attribute cs, $v_{i}$ represents the latent factors of item $i$ for its extrinsic characteristics, $d_{c}$ represents the latent factors of category c, and $q_{w}$ represents the latent factors of review word Moreover, Literal proposed a ranking-based geographical factorization technique for POI tips that obtains user-preference scores and geographical neighbor scores through user-POI matrix factorization and POI-k-nearest neighbor matrix factorization. Considered sequential influence, the place the next POI is influenced by the cutting-edge POI within a quick duration and the geographical impact of a far away POI is less possibly to be recommended, and proposed a personalized ranking metric that embeds a model for the next new POI recommendation.

\subsubsection{Geographical Area}

Apart from geographical neighbors, geo -graphical location is some other geographical affect that is used in a latent thing model. Many researchers [10-16] have currently observed spatial clustering phenomena in human mobility behavior and proven its effectiveness in POI recommendations.

For example, Liu et al. [14] proposed a novel place suggestion method that exploits instance-level traits and area -level traits by incorporating two level geographical characteristics into the learning of the latent factors of customers and locations.

The estimated ranking and objective function were:

$$
\begin{gathered}
\hat{r}_{u i}=\alpha U_{u} L_{i}+(1-\alpha) \frac{1}{Z\left(l_{i}\right)} \sum_{l_{i \epsilon} N\left(l_{i}\right)} \operatorname{Sim}\left(l_{i}, l_{1)} U_{u} L_{k}^{T}\right. \\
\min _{U, L} \frac{1}{2} \sum_{u, i}\left(r_{u i-} \hat{r}_{u i}\right)^{2}+\frac{\lambda_{1}}{2}\left(\|U\|_{F}^{2}\right)+\frac{\lambda_{2}}{2}\left(\|L\|_{F}^{2}\right) \\
+\lambda_{3} \sum_{g=1}^{G} \sum_{d=1}^{r} w_{g}\left\|L_{(g)}^{d}\right\|^{2}
\end{gathered}
$$

Where $\alpha \in[0,1]$ is the occasion weighting parameter, $N$ (li) is the set of $\mathrm{N}$ nearest neighboring places of $\mathrm{li}, \mathrm{N}(\mathrm{li})=\sum \mathrm{l} \in \mathrm{N}(\mathrm{li})$ $\operatorname{Sim}(\mathrm{li}, \mathrm{lk}), \operatorname{Sim}(\mathrm{li}, \mathrm{lk})$ is a Gaussian function, $\mathrm{L}_{(\mathrm{g})}^{\mathrm{d}} \in \mathrm{R}^{\mathrm{ngX1}}$ column vector in $\mathrm{L}(\mathrm{g})$, and $\mathrm{w}(\mathrm{g})$ is the weight assigned to $\mathrm{L}_{(\mathrm{g})}^{\mathrm{d}}$. Furthermore, leveraged a latent region variable to model consumer mobility behaviors over one of kind pastime regions and proposed a geographical probabilistic factor mannequin for POI recommendations. [16] proposed a probabilistic latent model by using considering the cluster phenomenon the place the users' check-in places were routinely divided into numerous regions, and how users' psychological conduct ought to make them decide on a nearby location to a far-off one.
The primary task to latent component mannequin is to comprise the geographical statistics into latent thing and minimize computational complexity.

\section{Geo-Social Information-Based User Model- ing}

Besides geographical and temporal influences, social have an impact on is any other source of contextual statistics exported to consumer modeling for POI recommendations. [27]

Check-ins in LBSNs exhibit $[1,23]$ that users long-distance journey is influenced through their buddies and customers are extra probable to visit locations that have been visited by way of their friends.

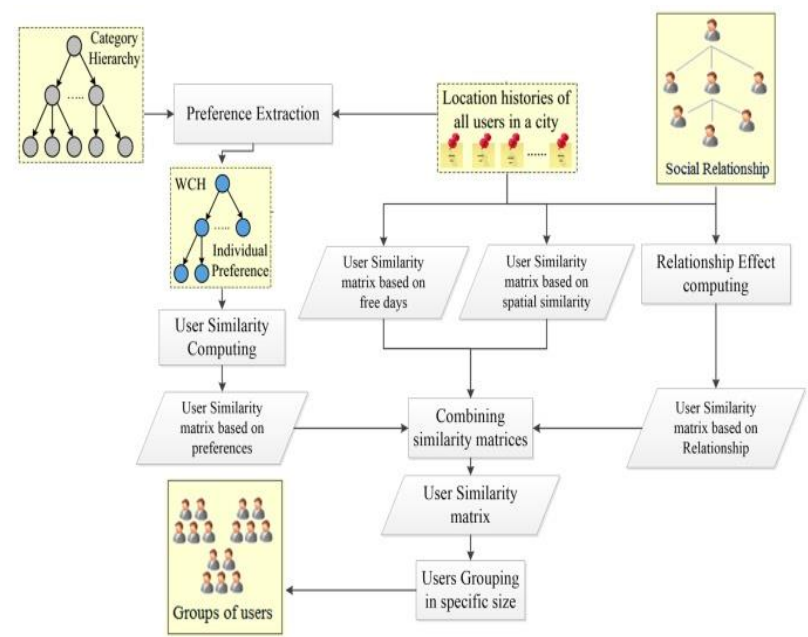

Fig. 4: The spatial and social relationships between users' check-ins in LBSNs.

In other words, buddies have a tendency to share more common pastimes than non-friends in LBSNs (the geographical and social relationships of users' check-ins in LBSNs. Their observations are widely exploited to mannequin users for POI pointers [2, 12-17]. For example, [12] proposed a Top-N POI advice algorithm through leveraging each the social and subject aspects of users' check-ins. [13] leveraged the location, time and social data to mannequin users and weighted about ranked pair wise losses to acquire top-n POI recommendations. [14] described a number of elements to measure the have an impact on of friends, rank buddies via a sequential random stroll with a restart in phrases of their influence, and utilized a Bayesian model to symbolize the dynamics of friends' have an effect on to predict locations. [15] Centered on the problem of predicting users' social have an effect on match pointers in event-based social networks and proposed a hybrid collaborative filtering mannequin through incorporating each event-based and user-based neighborhood influences into matrix factorization.

In addition to usual advice structures (e.g. e-commerce advice systems and context-aware advice systems), POI recommendation structures in LBSNs also face many challenging problems such as the issue of statistics sparsity and the user/POI cold-start problem. Incorporating social network ties into certain mathematical models (e.g. matrix factorization, design model) is a high-quality solution to cope with such challenges [18-23]. For example, [18] proposed a local event advice strategy that took Bayesian Poisson factorization as its simple unit to mannequin events, social relations, and content material text, and treated the cold-start hassle by way of incorporating match textual content and area statistics into these basic units. POI recommendation accuracy can be accelerated and cold-start problems can be addressed by leveraging the data of friends; $\mathrm{Li}$ et al. [18] first described three sorts of friend (social friends, place friends, and neighboring friends), and included the set of areas that obtained person likes and were checked-in by 
individual's buddies into matrix factorization. The estimated components and objective characteristic were as follows.

$\hat{p}_{u}=\left(q_{u i}+\varepsilon\right) U_{u}^{T} V_{i}$

$\underset{U, V, Q}{\arg \min } \sum_{u} E_{u}\left(p_{u i,} p_{u k,} p_{u h,} \hat{p}_{u i,} \hat{p}_{u k,} \hat{p}_{u h}\right)+\frac{\lambda_{u}}{2}\left(\|U\|_{2}^{2}\right)+\frac{\lambda_{v}}{2}\left(\|V\|_{2}^{2}\right)$ $+\frac{\lambda_{q}}{2}\left(\|Q\|_{2}^{2}\right)$

$\forall_{i} \in M_{u}^{0}, \forall_{k} \in M_{u}^{p}, \forall_{h} \in M_{u}^{w}$

Where $\mathrm{C} \in \mathrm{Q}$ is the class of place $\mathrm{I}$ and $\varepsilon$ is a tuning parameter. For every person $\mathrm{u}, M_{u}^{o}$ represents observed locations, $M_{u}^{p}$ conceivable locations $M_{u}^{w}$ other unobserved locations, and $E_{u}($.$) is the$ loss features for the observed, potential, and unobserved preferences of consumer $u$ for a location. Moreover, [14] exploited the geographical, social data and aspects extracted from person reviews to higher model user preferences, then built a novel heterogeneous plan by way of fusing three kinds of nodes (users, POIs and aspects) and a range of members of the family amongst them, ultimately changed the personalized POI recommendation as a sketch node ranking problem. [26]These processes may want to resolve person cold-start problem and alleviate the sparseness of users' check-ins with the aid of leveraging social information in LBSNs. The important task is how to comprise users' social family members into the popular models (such as matrix factorization).

\section{Conclusion}

The increasing use of smart devices and LBSNs has led to hundreds of thousands of user-generated records in recent years, how these facts can be utilized to understand human mobile conduct and help users make correct selections has attracted the pastime of many researchers from exclusive domains. In this paper, we have centered on reviewing the taxonomy of consumer modeling for POI recommendation by way of statistics evaluation in LBSNs. We have divided user modeling algorithms into four classes according to which kind of LBSNs facts has been wholly utilized in consumer modeling methods for POI recommendation: pure check-in data-based person modeling, geographical informationbased consumer modeling, spatio-temporal information-based consumer modeling, and geo-social information-based person modeling.

\section{References}

[1] Gao H, Tang J, Hu X \& Liu H, "Content-Aware Point of Interest Recommendation on Location-Based Social Networks", $A A A I$, (2015), pp.1721-1727.

[2] Wei LY, Zheng Y \& Peng WC, "Constructing popular routes from uncertain trajectories", Proceedings of the 18th ACM SIGKDD international conference on Knowledge discovery and data mining, (2012), pp.195-203.

[3] Liu H, Wei LY, Zheng Y, Schneider M \& Peng WC, "Route discovery from mining uncertain trajectories", IEEE 11th International Conference on Data Mining Workshops (ICDMW), (2011), pp.1239-1242.

[4] Yelp. Challenge Data Set. http://www.yelp.com/dataset_challenge, (2014).

[5] Zhang JD \& Chow CY, "iGSLR: personalized geo-social location recommendation: a kernel density estimation approach", 21 st ACM SIGSPATIAL International Conference on Advances in Geographic Information Systems, (2013), pp.334-343.

[6] Zhang JD \& Chow CY, "CoRe: Exploiting the personalized influence of two-dimensional geographic coordinates for location recommendations", Information Sciences, Vol.293, (2015) pp.163-181.

[7] Zhang JD \& Chow CY, (2015), “GeoSoCa: Exploiting geographical, social and categorical correlations for point-of-interest recommendations", Proceedings of the 38th International ACM SIGIR Conference on Research and Development in Information Retrieval, pp.443-452.
[8] Zhang JD \& Chow CY.. Spatiotemporal sequential influence modeling for location recommendations: A gravity-based approach. ACM Transactions on Intelligent Systems and Technology (TIST), Vol.7, No.1, (2015), pp.11-25.

[9] Zhang JD \& Chow CY, "TICRec: A probabilistic framework to utilize temporal influence correlations for time-aware location recommendations", IEEE Transactions on Services Computing, Vol.9, No.4, (2016), pp.633-646.

[10] Zhang JD, Chow CY \& Li Y, "Lore: Exploiting sequential influence for location recommendations", Proceedings of the 22nd ACM SIGSPATIAL International Conference on Advances in Geographic Information Systems, 2014, pp.103-112.

[11] Zhang JD, Chow CY \& Li Y, "iGeoRec: A personalized and efficient geographical location recommendation framework", IEEE Transactions on Services Computing, Vol.8, No.5, (2015), pp.701-714.

[12] Zhang JD, Chow CY \& Zheng, Y, “ORec: An opinion-based pointof-interest recommendation framework", Proceedings of the 24th ACM International on Conference on Information and Knowledge Management, (2015), pp.1641-1650.

[13] Cho E, Myers SA \& Leskovec J, "Friendship and mobility: user movement in location-based social networks", 17th ACM SIGKDD international conference on Knowledge discovery and data mining, (2011), pp.1082-1090.

[14] Rhee I, Shin M, Hong S, Lee K, Kim SJ \& Chong S, "On the levywalk nature of human mobility", IEEE/ACM transactions on net working (TON), Vol.19, No.3, (2011), pp.630-643.

[15] Gao H, Tang J, Hu X \& Liu, H, "Modeling temporal effects of human mobile behavior on location-based social networks", 22nd ACM international conference on Conference on information \& knowledge management, (2013), pp.1673-1678.

[16] Rahimi SM, Wang X \& Far B, "Behavior-based location recommendation on location-based social networks", Pacific-Asia Conference on Knowledge Discovery and Data Mining, (2017), $273-$ 285.

[17] Bahir E \& Peled A, "Identifying and tracking major events using geo-social networks", Social science computer review, Vol.31, No.4, (2013), pp.458-470.

[18] Xu Z, Liu Y, Yen N, Mei L, Luo X, "Crowd sourcing based description of urban emergency events using social media big data", IEEE Transactions on Cloud Computing (TCC), Vol.9, No.9, (2016), pp.1-1.

[19] Xu Z, Liu Y, Xuan J, Chen H \& Mei L, "Crowd sourcing based social media data analysis of urban emergency events", Multimedia Tools and Applications, Vol.76, No.9, (2017), pp.11567-11584.

[20] Yin J, Lampert A, Cameron M, Robinson B \& Power R, "Using social media to enhance emergency situation awareness", IEEE Intelligent Systems, Vol.27, No.6, (2012), pp.52-59.

[21] Shen Y \& Karimi K, "Urban function connectivity: characterisation of functional urban streets with social media check-in data", Cities, Vol.55, (2016), pp.9-21.

[22] Zhou X, Hristova D, Noulas A \& Mascolo C, "Detecting socioeconomic impact of cultural investment through geo-social networks analysis", 11th international AAAI Conference on Web and Social Media(ICWSM), Montreal Canada, (2017), pp.15-18.

[23] Zhu WY, Peng WC, Chen LJ, Zheng K \& Zhou X, "Modeling user mobility for location promotion in location-based social networks", 21th ACM SIGKDD International Conference on Knowledge Discovery and Data Mining, (2015), pp.1573-1582.

[24] Cheng C, Yang H, King I \& Lyu MR, "A unified point-ofinterest recommendation frame -work in location-based social networks", ACM Transactions on Intelligent Systems and Technology (TIST), Vol.8, No.1, (2016).

[25] Sai Prasad K, Chandra Sekhar Reddy N, Rama, B, Soujanya A \& Ganesh D, "Analyzing and Predicting Academic Performance of Students Using Data Mining Techniques", Journal of Advanced Research in Dynamical and Control Systems, Vol.10, No.7, (2018), pp.259-266.

[26] B Kassimbekova, G Tulekova, V Korvyakov (2018). Problems of development of aesthetic culture at teenagers by means of the Kazakh decorative and applied arts. Opción, Año 33. 170-186

[27] M Pallarès Piquer and O Chiva Bartoll (2017). La teoría de la educación desde la filosofía de Xavier Zubiri. Opción, Año 33, No. 82 (2017): 91-113 